\title{
Development of a chopper charge amplifier for measuring the cavity pressure inside injection moulding tools and signal optimisation with a Kalman filter
}

\author{
Manuel Schneider ${ }^{1}$, Alexander Jahn ${ }^{1}$, Norbert Greifzu ${ }^{1}$, and Norbert Fränzel ${ }^{1,2}$ \\ ${ }^{1}$ Faculty of Electrical Engineering, Schmalkalden University of Applied Sciences, Blechhammer 9, \\ 98574 Schmalkalden, Germany \\ ${ }^{2}$ Advanced System Technology (AST) Branch of Fraunhofer IOSB, Am Vogelherd 50, \\ 98693 Ilmenau, Germany \\ Correspondence to: Manuel Schneider (m.schneider@hs-sm.de)
}

Received: 12 August 2016 - Revised: 7 April 2017 - Accepted: 12 April 2017 - Published: 10 May 2017

\begin{abstract}
This article provides insight into the development of a powerful and low-cost chopper amplifier for piezoelectric pressure sensors and shows its possible applications for injection moulding machines. With a power supply of 3.3 volts and the use of standard components, a circuit is introduced which can be connected to a commercially available microcontroller without any additional effort. This amplifier is specialised for low frequencies and high-pressure environments. With the adjustment of the sample and chopper frequency by means of software, the amplifier can easily be adapted for other applications. This chopper amplifier is a very compact and cost-effective solution with a small number of required components. In this contribution, it will be shown that the amplifier has good results in various laboratory tests as well as in the production process. Furthermore, an approach to fuse data from force and pressure signals by using a Kalman filter will be presented. With this method, the quality of the sensor signals can be significantly improved. This article is an extension of our previous work in Schneider et al. (2016b).
\end{abstract}

\section{Introduction}

Two key factors for the pricing of injection-moulded articles are the consistent quality of the production and the production volume. Today, production parameters are recorded and evaluated by means of machine-specific hardware outside the injection mould. The measured data are then available for the machine or the SCADA ${ }^{1}$. This requires that all machines are equipped with special hardware and that the company has the necessary infrastructure available at the plant. Current systems keep the parameterisation data for the production process in memory located inside the control unit of the moulding machine or on compact discs. In the case of location changes, e.g. installation by the tool manufacturer at the

\footnotetext{
${ }^{1}$ System for supervisory control and data acquisition (SCADA).
}

manufacturing site or a change in the manufacturing plant, the tuned injection moulding parameters are often lost. One possible solution could be the use of an online data management system, but this causes a lot of other problems with data security, violating intellectual property or company espionage.

Another possibility could be to incorporate an embedded system into the injection moulding tool. This technology would enable the system to keep track of all activities in the production process. Following this principle, one can think of a system that locally stores all data related to the whole life cycle of a moulding tool and also ensures that companies can keep their knowledge confidential. This EDS ${ }^{2}$ enables independent quality monitoring and thus ensures the

\footnotetext{
${ }^{2}$ Embedded diagnostics system (EDS).
} 
security of the IT infrastructure. It can also be used by companies without a SCADA system or without a connection to the World Wide Web. This paper discusses a measurement technique that is suitable for sampling pressure data with low power consumption while being accurate enough to compete with existing industrial solutions. The TLV2771 (Texas Instruments, Dallas, TX, USA) is a standard operational amplifier for charge amplification. This $\mathrm{IC}^{3}$ is only usable with a single supply when the sensors are connected to $3.3 \mathrm{~V}$ and to the reference voltage. In our case, the piezoelectric sensors always have a connection to the machine ground, and this is a problem for the amplifier. The developed amplifier shows an alternative method of single-supply charge amplification with a ground connection. The contribution is structured as follows. Sections 2 and 3 give an overview of the system specifications and the basics of the developed chopper charge amplifier. In Sect. 4, the focus is on the simulation of the chopper amplifier and the verification of the simulation results. The temperature behaviour and the amplifier quality are the main aspects in Sect. 5. Information on the injection moulding process and the results of the measurement in the production process can be found in Sect. 6. In Sect. 7, the focus is on the signal optimisation by using a Kalman filter. The conclusion is the last part of this contribution.

\section{System specifications}

For use within injection moulding machines, two companies are developing industrial charge amplifiers for pressure measurement. The voltage specifications, the dimensions and the price of commercial charge amplifiers from those companies are not within our specifications. Two examples of these amplifiers are the 5073Axx1 (Kistler Group, 2012) and the 5050A (Priamus System Technologies GmbH, 2015). In Table 1 , some of the technical information for these two charge amplifiers is listed.

The goal of this work is to propose a new charge amplifier with a low supply voltage, a symmetrical output signal and small dimensions. The following list contains the requirements for the development of this charge amplifier.

1. A regulated power supply with $\mathrm{V}_{\mathrm{dc}}=3.3 \mathrm{~V}$ should be used.

2. The output signals must be in the range of $0 \ldots 3.3 \mathrm{~V}$ for optimal analogue-to-digital conversion.

3. The occupied space must be kept as small as possible.

4. The signal quality must be better than the indirect measurement with a force sensor.

5. The cost of the amplifiers must be kept low.

\footnotetext{
${ }^{3}$ Integrated circuit (IC)
}

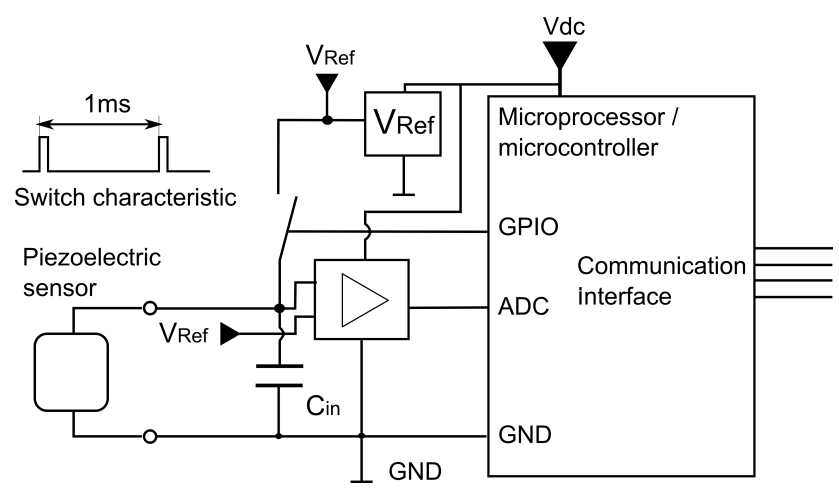

Figure 1. A diagram of the chopper amplifier with its main components and a connected microcontroller, the piezoelectric sensor and the timing of the electric switch.

\section{Basics for the chopper charge amplifier}

To meet the requirements of space, power and cost minimisation, the authors developed and evaluated different charge amplifier concepts. In most cases, the insulation of the $\mathrm{PCB}^{4}$ and the selected electrical components is too low, and the charge signal is rapidly lowered to zero. The first acceptable result has been achieved with a chopper amplifier. This amplifier structure is developed according to Enz and Temes (1996). The amplifier operates with a clocked reference voltage source at the input capacitor and thus enables the measurement of charge changes in the positive and negative direction with respect to the reference voltage (Jahn, 2015). The reference voltage is generated by an integrated circuit (REF3212; Texas Instruments). A CMOS ${ }^{5}$ switch is used to reset the electrical charge to the reference voltage on the input capacitor. The reset frequency can be adjusted with software and is $1 \mathrm{kHz}$. The two main components of the chopper amplifier are an instrumentation amplifier and a microcontroller. The microcontroller TM4C123G (Texas Instruments) has a 12-bit $\mathrm{ADC}^{6}$ input and a communication interface. The piezoelectric signal is sampled by the ADC before every reset impulse. In Fig. 1, the schematic circuit diagram of the developed chopper amplifier is shown. In this special solution, the isolation effect of the PCBs and the cost of the PCB design could be reduced. In Fig. 2, the developed chopper amplifier without the microcontroller is displayed.

By using a pulsed reference voltage source, the signal of interest is approximately differentiated. Eqs. (1) and (2) reflect the mathematical and temporal relation at the input capacitance of the chopper amplifier. For case (a), the voltage at the input capacitance approaches the voltage of $U_{\text {ref. }}$ In case (b) when the time $t$ is between two resets, the voltage at the capacitance is a function of the charge output from the piezo-

\footnotetext{
${ }^{4}$ Printed circuit board (PCB)

${ }^{5}$ Complementary metal-oxide-semiconductor (CMOS)

${ }^{6}$ Analogue-to-digital converter (ADC)
} 
Table 1. The technical data for the 5073Axx1 (Kistler Group, 2012) and the 5050A (Priamus System Technologies GmbH, 2015) charge amplifiers.

\begin{tabular}{lcrl}
\hline Characteristic & 5073Axx1 & $5050 \mathrm{~A}$ & Physical unit \\
\hline Supply voltage & $18-30$ & $15-30$ & $V_{\mathrm{dc}}$ \\
Measuring range & $\pm 100 \ldots \pm 1000000 \quad \pm 5000 \ldots \pm 100000$ & $\mathrm{pC}$ \\
Output voltage & $-10 \ldots+10$ & $\mathrm{~V}$ \\
Operating temperature range & $0 \ldots 60$ & ${ }^{\circ} \mathrm{C}$ \\
Height & 64.0 & $\mathrm{~mm}$ \\
Length & 115.0 & $\mathrm{~mm}$ \\
Width & 34.5 & $\mathrm{~mm}$ \\
\hline
\end{tabular}

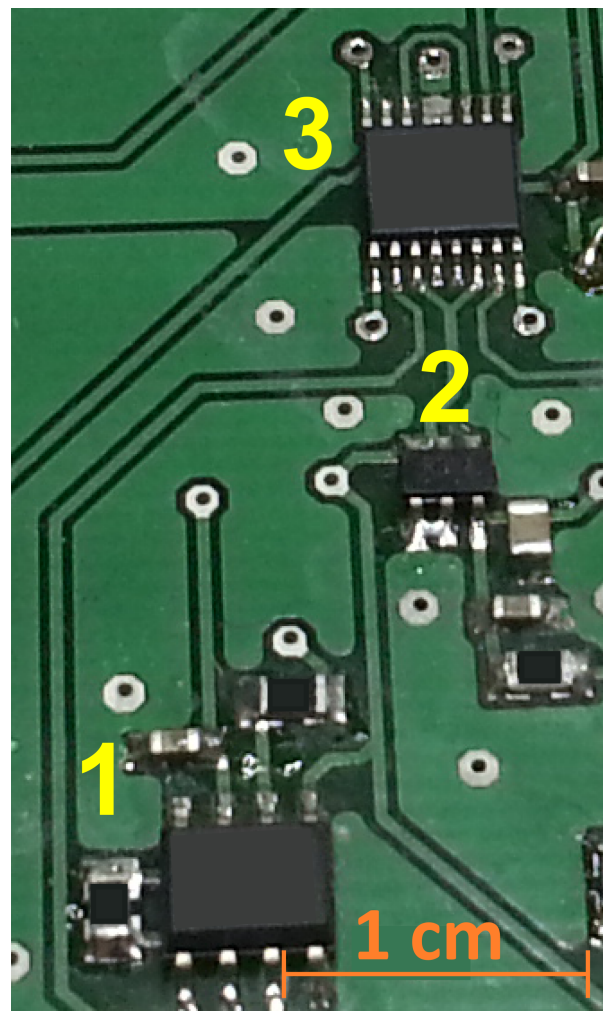

Figure 2. A picture of the developed chopper amplifier with the associated board cut-out (Schneider et al., 2016a). The components in the picture are 1 . the instrumentation amplifier, 2. the reference voltage source and 3. the electric switch.

electric sensor. In Eq. (3), the discharge curve during the reset interval of case (a) is described. The $R$ is the input resistance of the circuit. In our case, $R$ is made up of the input resistance of the CMOS switch and is greater than $10 \mathrm{M} \Omega$. The $C$ is the input capacitance of the circuit additional to the cable capacitance. In our case, the cable length is shorter than $30 \mathrm{~cm}$, and thus the equation can be simplified to $C=C_{\text {in }}$. The value of $C_{\text {in }}$ is the capacitor value of the amplifier input and has to be adjusted to the PCB design:

$$
u_{\left(C_{\text {in }}\right)}(t)= \begin{cases}U_{\text {ref }}(t), & \text { case (a) } \\ \frac{Q_{\text {piezo }}(t)}{C_{\text {in }}}+U_{\text {ref }}(t), & \text { case (b) }\end{cases}
$$

(a) $(n+1) \cdot \Delta t-t_{\text {reset }}<t<(n+1) \cdot \Delta t$

(b) $n \cdot \Delta t<t<(n+1) \cdot \Delta t-t_{\text {reset }}$

$(n \in \mathbf{N})$ - sample number,

$$
Q_{\text {piezo }}(t)=\int_{t+\Delta t-t_{\text {reset }}}^{t+\Delta t} i_{\text {piezo }}(t) \mathrm{d} t,
$$

for a) $u_{\left(C_{\text {in }}\right)}(t)=U_{\text {ref }}\left(1-e^{-\frac{t}{R \cdot C}}\right)+U_{\text {start }}(t)$.

The voltage $U_{\text {start }}(t)$ from Eq. (3) describes the voltage before every reset. At the first start of the chopper amplifier, the start voltage $U_{\text {start }}(t)$ is equal to zero. At every following reset, however, the value of $U$ from Eq. (4) changes between zero and the supply voltage. This results in a charge curve, or a discharge curve, to the reference voltage:

$U_{\text {start }}(t)=U\left(e^{-\frac{t}{R \cdot C}}\right)$.

This charging takes place in finite time. That means that during the reset time, the accumulated charge signal cannot be measured, thus leading to incorrect measurements of the charge signal. To keep the information loss as low as possible, $t_{\text {reset }}$ should be much smaller than the sample time $\Delta t$. However, this time depends on the input capacitor, the PCB isolation and the leakage current of the other electrical components on the PCB. According to Jahn (2015), a value of $100 \mathrm{pF}$ requires $400 \mathrm{~ns}$ for a complete discharge from $U_{\text {Ref }}$ to ground by using the ADG612 (Analog Devices, Massachusetts, USA) analogue switch. In this case, it could be used as a benchmark for the reset time of the chopper amplifier. Fig. 3 shows one result of the charge and discharge curve of such a $100 \mathrm{pF}$ capacitor. The orange line is the $400 \mathrm{~ns}$ charge impulse and the blue line describes the charge and discharge curve of the input capacity.

The discharge time depends on the measurement set-up with the oscilloscope and the analogue switch. In this experiment, the shortest switching pulse of the analogue switch is 


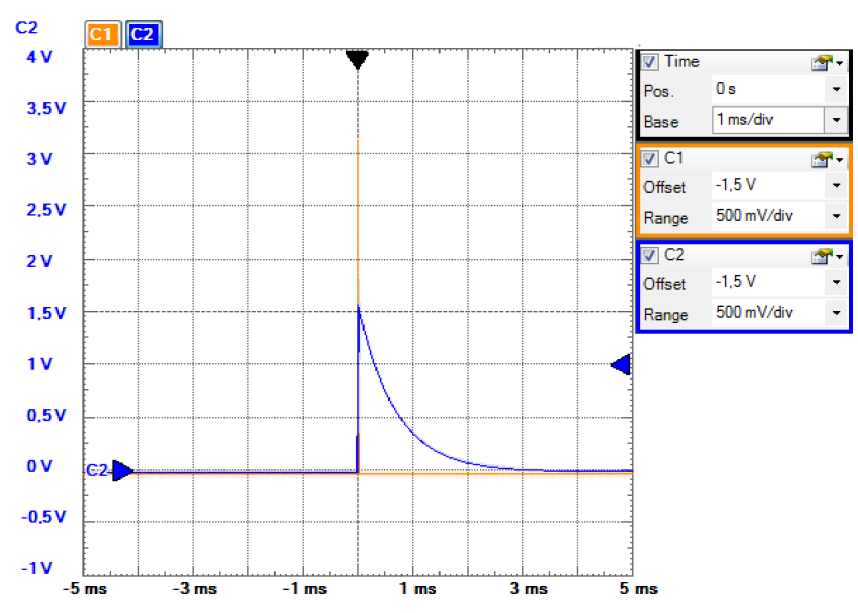

Figure 3. The result of the shortest switching pulse from the analogue switch ADG612. A pulse of $400 \mathrm{~ns}$ (orange curve) is enough to load the capacitor from zero to the reference voltage. After the pulse, the voltage returns to zero in a time of approximately 4 milliseconds (blue curve; Jahn, 2015).

determined. In the next section, the operation of the chopper amplifier is verified by a simulation.

\section{Simulation of the chopper charge amplifier}

\subsection{Basics of the chopper simulation}

To illustrate the operation of this amplifier, a simulation script in $\mathrm{SCILAB}^{7}$ has been written. The basis of this simulation is a periodic signal, which is passed as a voltage signal to the input of the chopper amplifier. For the simulation, a signal with two constant derivation values was chosen. The simplest signal with these characteristics is the triangular wave. The advantages of this signal are the two different derivation values and the numbers of different Fourier frequencies. In Eq. (5), the analytical expression of this test signal in the time interval from $t=t_{0}=0 \mathrm{~s}$ to $t=T$ ( $T$ is the sampling interval of the signal) is shown. The SCILAB plot in Fig. 4 shows the triangular signal. Another method to generate a triangular test signal is the development of a Fourier series. In Eq. (6), the result of the Fourier series development for the periodic test signal is displayed. The variable $\hat{u} / 2$ stands for the maximum value of the set voltage. Both equations represent the functional relationship between voltage and time. The variable $\omega_{0}$ represents the radian frequency of each oscillation. The result of the superposition of this Fourier function is the same as shown in Fig. 4 and Eq. (5). For this simulation, $\hat{u}$ is set to $\pi$ :

$u(t)=\left\{\begin{array}{ll}\frac{2 \cdot \hat{u}}{T} \cdot t, & \text { case (a) } 0 \leq t \leq \frac{T}{2} \\ -\frac{2 \cdot \hat{u}}{T} \cdot t+\hat{u}, & \text { case (b) } \frac{T}{2} \leq t<T\end{array}\right.$,

\footnotetext{
${ }^{7} \mathrm{SCILAB}$ is an open-source software for numerical computation; http://www.scilab.org.
}

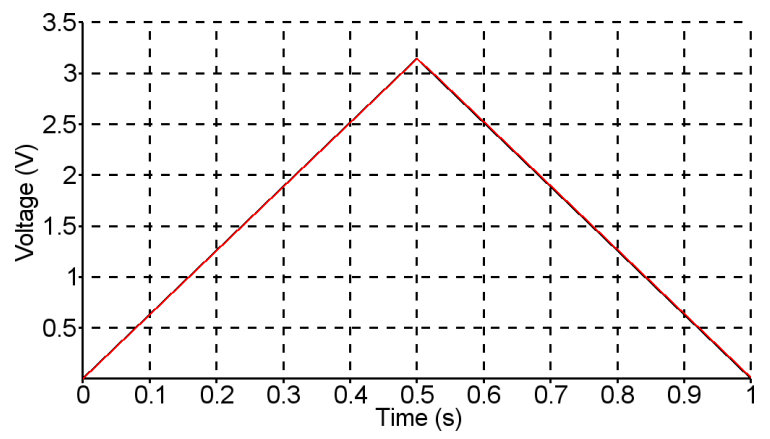

Figure 4. A representation of the triangular test signal with an interval of $T=1 \mathrm{~s}$. The line shows the input signal, and the same signal is the result of the integration of the digital sampled chopper signal from Fig. 5.

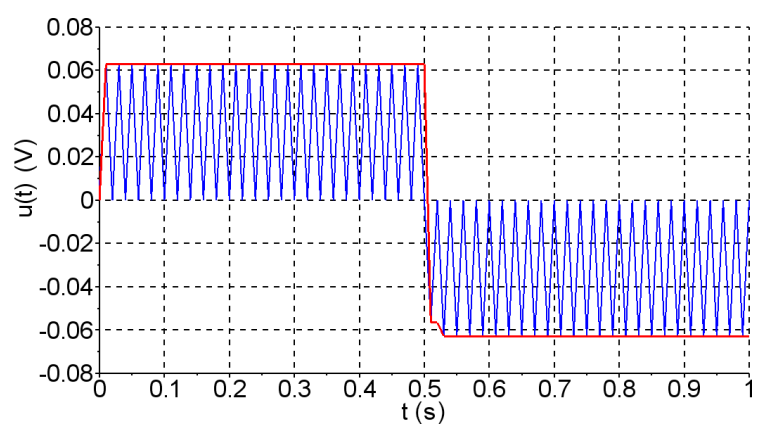

Figure 5. The blue signal illustrates the simulated chopper output signal with a sampling frequency of $100 \mathrm{~Hz}$. The red line illustrates the sampled values of the blue time signal.

$$
u(t)=\frac{\hat{u}}{2}-\frac{16 \cdot \hat{u}}{\left(\omega_{0} T\right)^{2}}\left(\frac{\cos \left(1 \omega_{0} t\right)}{1^{2}}+\frac{\cos \left(3 \omega_{0} t\right)}{3^{2}}+\cdots\right) .
$$

In Fig. 5, the operation of the chopper amplifier is illustrated. The capacitor voltage is periodically set to zero (here $T=10 \mathrm{~ms}$ ). This results in the characteristic chopped signal, which is amplified further. It can be seen that the chopper amplifier generates an approximation of the derivative of the input signal. To reduce the number of plotting lines in the simulation, the chopper frequency is set to $100 \mathrm{~Hz}$.

This signal is sensed and converted into digital values. The optimal result of the derivative triangular signal is a rectangular signal. In order to restore the input signal from the digital values, the digitised samples must be integrated. Through the integration of the chopper signal, the test signal (triangular signal) is formed again. The result is the same curve from Fig. 4. These simulation results are also verified with the developed chopper amplifier. 


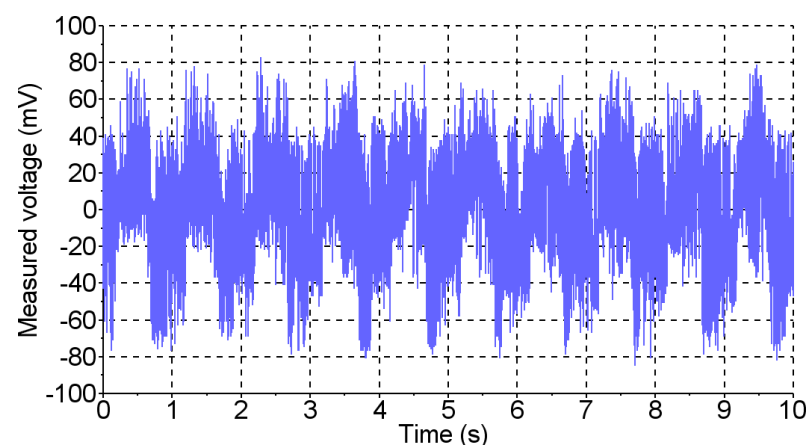

Figure 6. Display of the chopped input triangular signal after the amplifier (recording time $10 \mathrm{~s}$; peak-to-peak amplitude $\hat{u}$ at the signal generator $120 \mathrm{mV}$; time period $T=1 \mathrm{~s}$; amplifier gain 50).

\subsection{Verification of the simulation results}

The peak-to-peak amplitude of the laboratory triangular test signal is set to $120 \mathrm{mV}$ and the frequency is $1 \mathrm{~Hz}$. This test signal was generated by an function generator. The sampled, chopped and amplified triangular test signal is shown in Fig. 6. The expected rectangular oscillation is not clearly visible.

The distortion is mainly caused by the $50 \mathrm{~Hz}$ power grid frequency. In this experiment, the chopper frequency is set to $1 \mathrm{kHz}$. This resulted in a lower amplitude of the chopper signal, but with the gain value of 50 the signal could be measured. The effective voltage over $500 \mathrm{~ms}$ segments is calculated with $\approx \pm 12 \mathrm{mV}$. The main information of the test signal is smaller than the noise level.

To produce the original test signal, different mathematical operations have to be performed. The first is the subtraction of the reference voltage. The next step is the integration of the time signal, and the last is the division of the amplifier gain value. The result of the first two operations is displayed in Fig. 7. As can be seen in the illustration, the peaks in the signal increase with time. There is drift in the data. The reason could be an integration error or the interference signals of the chopper signal from Fig. 6. Another possible reason for the drift could be an unstable reference voltage. For long measurement times, an offset in the reference voltage results in a drift in the positive or negative direction.

As can be clearly seen, the amplified voltage level is approximately $6 \mathrm{~V}$ and the time period is $1 \mathrm{~s}$. With the division of the gain value, the result is approximately $120 \mathrm{mV}$.

In Sect. 7.2, a solution for the drift problem with long measurement times will be proposed.

\section{Measurement of the amplifier quality}

\subsection{Laboratory set-up for the resolution measurement}

For the study of the resolution measurement and the temperature behaviour of the amplifier, a test set-up using a temper-

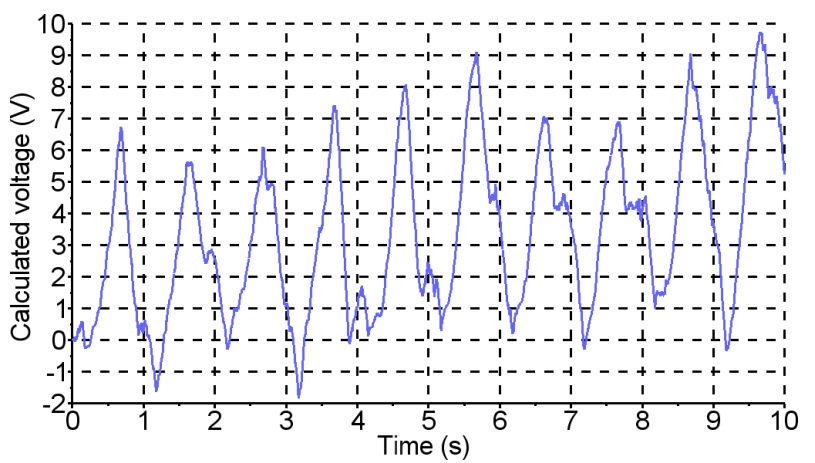

Figure 7. The integration result of the noisy test signal from Fig. 6.

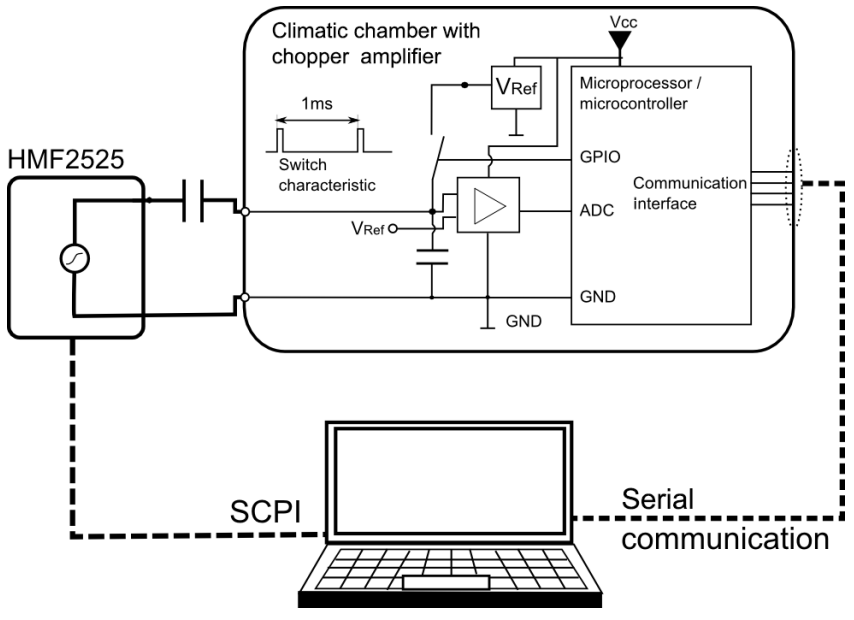

Figure 8. A schematic representation of a fully automated measurement set-up with a signal generator, amplifier circuit and data analysis with laptop and SCILAB.

ature controlled chamber, a signal generator and a computer system was developed. The computer system automatically controls the experiment properties of the HMF2525 signal generator (ROHDE \& SCHWARZ, Münich, Germany) and measures the signals from the chopper amplifier inside the climatic chamber. In Fig. 8, the measurement set-up is displayed.

For the evaluation, two typical temperatures were chosen that represent the expected conditions inside the mounted EDS. The set of ambient temperatures in the chamber were 25 and $50^{\circ} \mathrm{C}$. The computer sends the configuration for the HMF2525 signal generator via the standard $\mathrm{SCPI}^{8}$ interface and simultaneously receives the converted values of the $\mathrm{AD}$ converter. The recorded data are used to determine the temperature-dependent signal-to-noise ratio and the threshold assessment of the amplifier structure.

In order to determine the signal-to-noise ratio and the sensitivity of the developed charge amplifier, the fully automatic measurement set-up, as shown in Fig. 8, was used. Due to

\footnotetext{
${ }^{8}$ Standard Commands for Programmable Instruments (SCPI)
} 


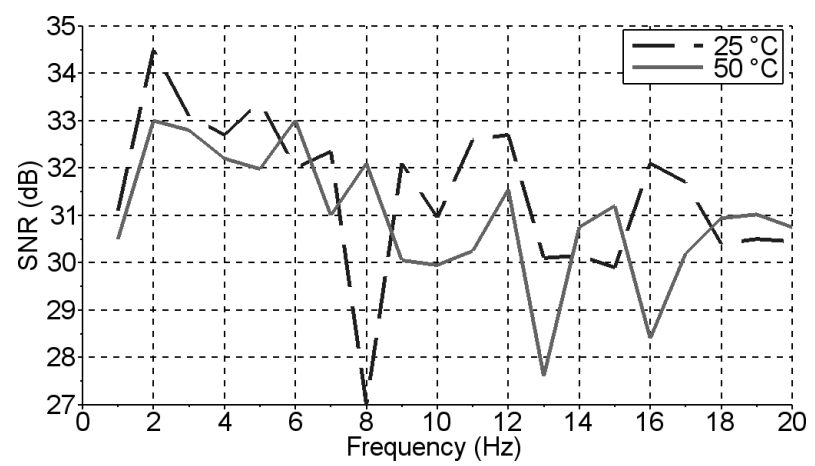

Figure 9. The measurement results for the signal-to-noise ratio subject to the input frequency and the ambient temperature as described in Enz and Temes (1996).

the fact that it is not typical to specify a signal-to-noise ratio in commercial charge amplifiers, additional information for this special amplifier is provided in this section. A number of sine waves with different frequencies were used as test signals. The series of measurements started at a frequency of $f=1 \mathrm{~Hz}$ and was performed in 19 steps up to $f=20 \mathrm{~Hz}$. The calculated values of the spectral coefficients of the input signal are divided by the sum of all the frequency components that are independent of the input signal to respectively calculate the signal-to-noise ratio. The mathematical relation for this is shown in Choi (2006).

The threshold assessment allows for a qualitative and quantitative assessment of the quality of the proposed charge amplifier. The threshold value of the circuit is the smallest measurable change in charge (in $\mathrm{pC}$ ) that produces a change in the least significant bit (LSB) of the sampled voltage signal. A rectangular oscillation voltage at the input of the amplifier is applied for this measurement. This rectangular oscillation passes the chopper amplifier and results in an alternating periodic needle pulse similar to Dirac pulses. In Sect. 5.2, the results of these measurements are described.

\subsection{Signal quality results of the chopper amplifier}

For the measurement of the signal-to-noise ratio, different sine waves with frequencies of 1 to $20 \mathrm{~Hz}$ were used. The calculated signal-to-noise ratio (SNR) is on average around $31.49 \mathrm{~dB}$ at $\mathrm{T} 1=25^{\circ} \mathrm{C}$ and around $30.96 \mathrm{~dB}$ at $\mathrm{T} 2=50^{\circ} \mathrm{C}$. The results of the noise measurements for different input frequencies are shown in Fig. 9. As can be seen in this measurement, there are three runaways at different frequencies. The reason could be the climatic chamber itself. There are some engines and electronics that disturb the measurement at varying times. At these times, the frequency disturbances in other frequency ranges increase and result in negative peaks in the measurements.

A solution for this problem can be a delayed start of the measurement after a settling time for the climatic chamber.

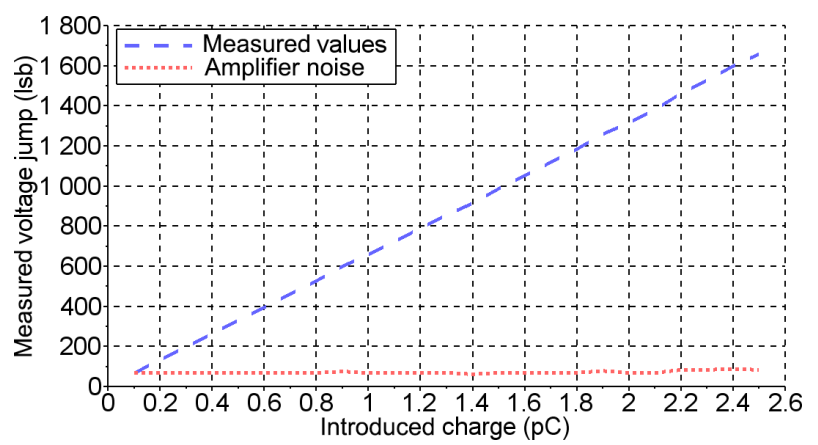

Figure 10. The threshold assessment (intersection point of the graphs at approximately $0.1 \mathrm{pC}$ ) of the circuit at $25^{\circ} \mathrm{C}$. The blue dashed line shows the peak voltage in the measurement data at a corresponding input charge. The red dotted line shows an increase in the calculated amplifier noise by 3 times, similar to Enz and Temes (1996).

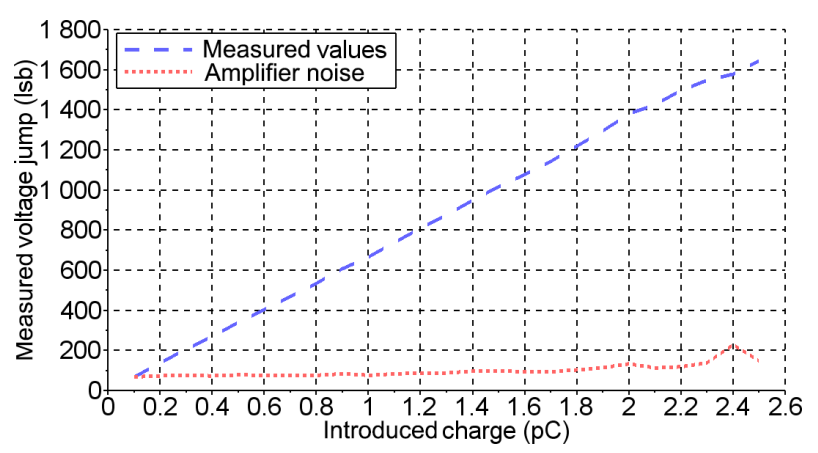

Figure 11. The threshold assessment (intersection of the graphs at approximately $0.12 \mathrm{pC}$ ) of the circuit at $50^{\circ} \mathrm{C}$ of ambient temperature. The blue dashed line shows the swing in the measurement data at a corresponding input charge. The red dotted line shows a threefold increase in the calculated amplifier noise, similar to Enz and Temes (1996).

The results of the threshold assessment measurement can be seen in Figs. 10 and 11. In this case, there is a linear relationship between the input signals and the measured output values. In both figures, the level of interference at different ambient temperatures is nearly constant.

As a basis for the calculation of the amplifier noise, the mean square of the noise is multiplied by a factor of 3 (Gautschi, 2002, p. 57). The threshold of the charge amplifier can be read at the intersection between the measured value (blue) and the amplifier noise (red). It is about $0.1 \mathrm{pC} / \mathrm{LSB}$; the optimisation of the resolution is part of further work. According to Gautschi (2002), commercial charge amplifiers have a threshold of $1 \mathrm{fC}$. The next section gives an overview of the measurement in the production process. 


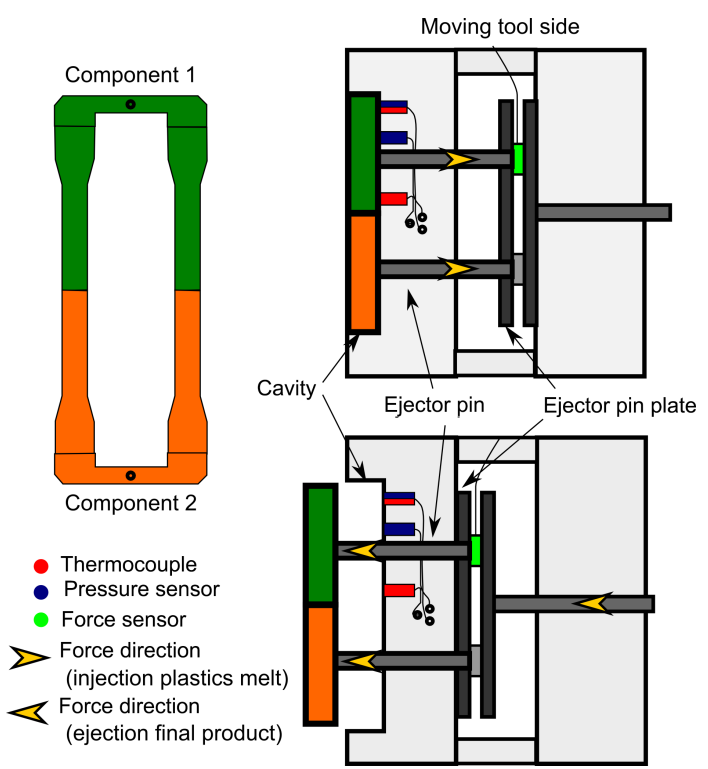

Figure 12. A 3-D model of the investigated tool with the different sensor types and their respective positions. The red points show the positions of the thermocouple sensors. The yellow points are the piezoresistive force sensors, also called load cells. The blue points are the piezoelectric pressure sensors, which are used for the measurement of the injection pressure of the molten plastics. A view of the cut through the plane is displayed in Fig. 13.

\section{Measurement during the production process}

\subsection{Information about the injection moulding process}

The purpose of this chopper amplifier is the measurement of pressure sensor signals inside the cavity during the injection moulding process. To get a better understanding of the injection moulding tool, it is necessary to give some information about it. The application for our sensor system is a twocomponent injection moulding tool. The tool produces a twocomponent multipurpose test specimen ${ }^{9}$ as specified in ISO 294-1 (1996). Additional information for the topic of polymer testing can be found in Grellmann and Seidler (2013, p.15) and Seefried and Drummer (2015). For the measurement of the production quality of the tensile bar, a range of sensors within the injection moulding tool is accommodated. The sensor positions and different sensor types are illustrated in Fig. 12.

For the temperature measurement of the flowing plastics, four thermocouples are inserted inside the cavity. Furthermore, two thermocouples are applied inside each tool half to measure the tool temperature. Four piezoelectric pressure sensors are inserted inside the cavity of the injection tool. They measure the injection pressure of the flowing plastics melt. The two load cells are placed between the ejector pins

\footnotetext{
${ }^{9}$ The multipurpose test specimen is also referred to as a tensile bar or test piece.
}

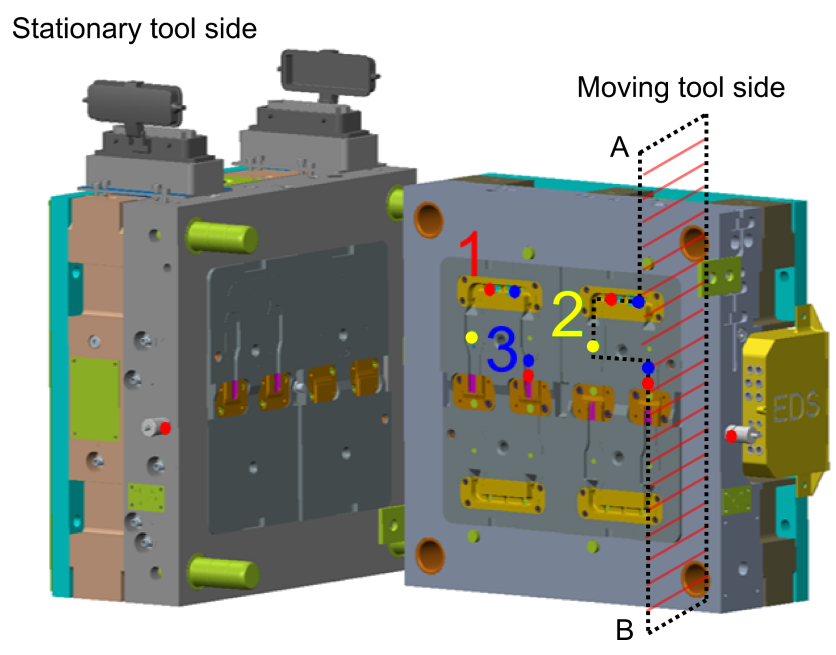

Figure 13. On the left side is a sketch of the produced twocomponent tensile bar. On the right side is a cross-section of the moulding tool to show the ejector and the sensor position.

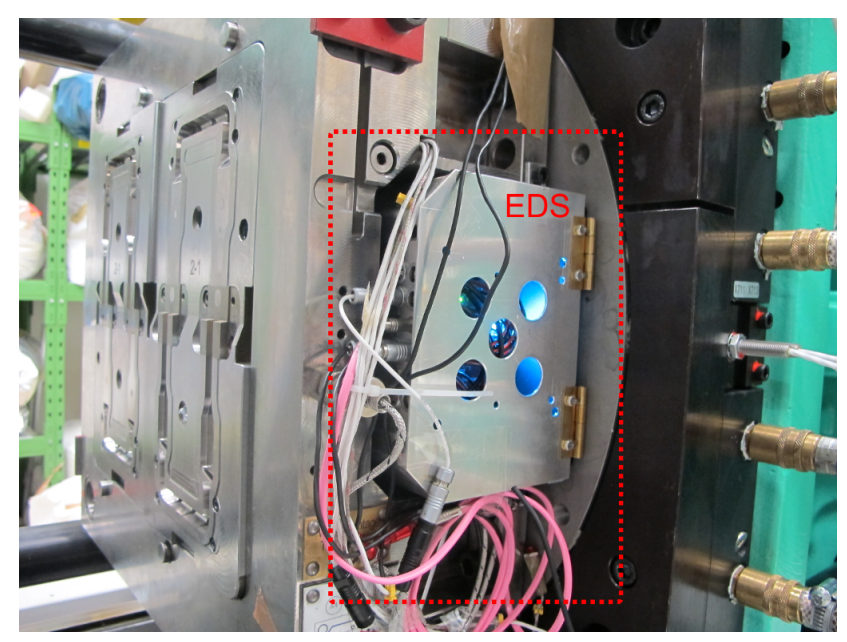

Figure 14. A photograph of the moving side of the two-component injection moulding tool. In the middle of the picture is the embedded measurement hardware (EDS; Schneider et al., 2016b).

and the ejector plate. They record the ejection force of the produced tensile bar after the cooling phase of the process. The secondary effect of this kind of sensor is an indirect injection pressure measurement. Figure 13 shows the produced test piece; on the right side is a cut through the moulding tool at the ejection step of the final product.

In Fig. 14, the injection moulding tool with mounted embedded measurement hardware (EDS) is shown.

\subsection{Measurement results during the manufacturing process}

In this section, the measurement results during the manufacturing process are described. The signal from the chopper 


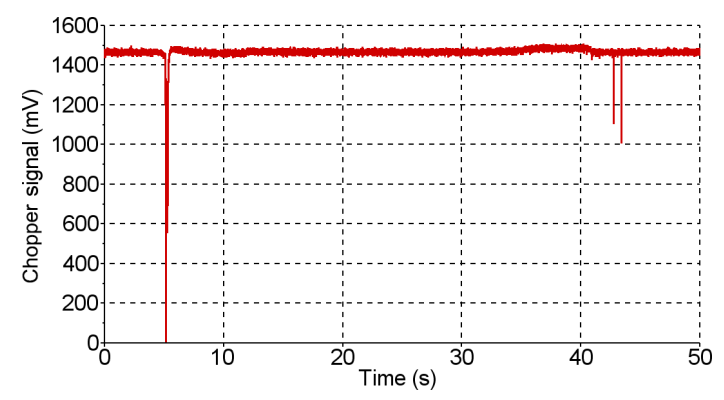

Figure 15. The sampled chopper signal positive pressure changes result in a downward-facing peak relative to the reference voltage (resulting pressure gradient at Priamus 6001A).

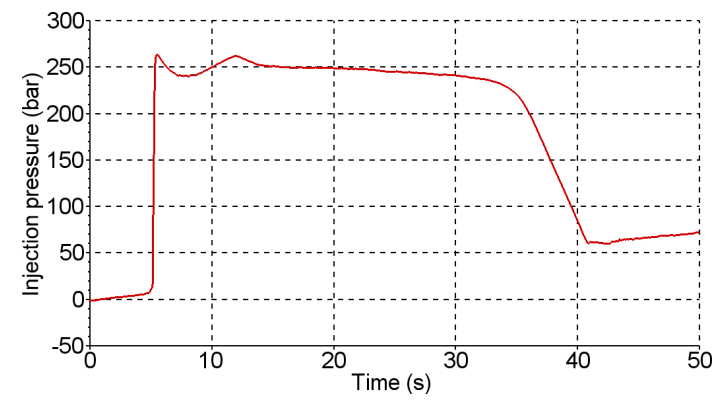

Figure 16. The results of the absolute value of the integrated chopper signal after the mathematical operations. It is the reconstructed injection pressure of the plastics melt into the cavity.

amplifier is shown in Fig. 15. The recording time was limited to $50 \mathrm{~s}$, which is the interval time for the manufacturing process of the produced tensile bar in the injection mould.

The negative modulation in the charge curve in Fig. 15 is due to the mechanical structure of the piezoelectric sensor. A inversion of the signal around the reference voltage can be achieved with software. With the mathematical operations described in Sects. 4.1 and 4.2, the pressure signal can be reconstructed. In Fig. 16, the results of the calculations and integration of the chopper signal are displayed. It illustrates one injection moulding process with a very low noise level.

For the validation of the pressure signal, a second sensor can be used. As shown in Fig. 13, the force sensors are in a good position to perform an indirect measurement of the injection pressure. The measured force signal of the same production cycle as in the previous pictures is displayed in Fig. 17.

Both measurement results from Figs. 16 and 17 have a similar shape. A major difference lies in the recorded noise levels of these sensors. The interference level from the force sensor is greater than that of the integrated pressure signal. Furthermore, a significant temporal difference between the sensor signals can be observed after $40 \mathrm{~s}$. The reason for that can be the different measurement positions of each sensor in the moulding tool. The greatest difference can be found in

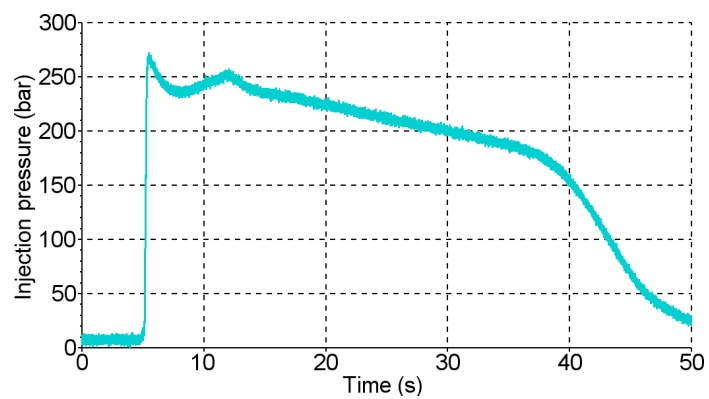

Figure 17. A diagram of the pressure curve at the KM26 force sensor.

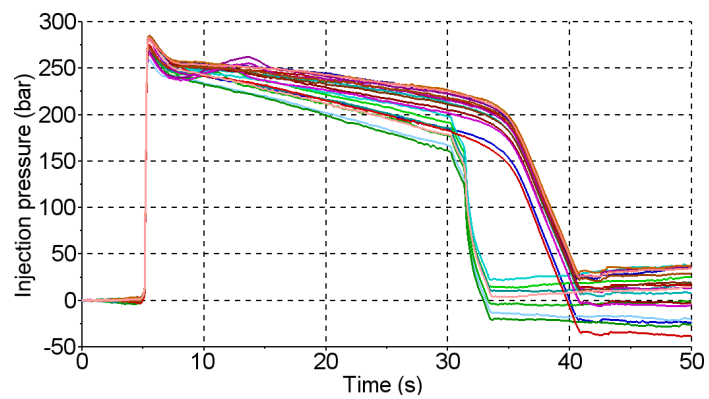

Figure 18. The 25 production intervals measured by the chopper charging amplifier; the interval time is $50 \mathrm{~s}$.

the drift of the integrated chopper signal. The reasons for that are described in Sect. 4.2.

In Fig. 18, the 25 piezoelectric pressure curves of the chopper charging amplifier are shown.

As can be seen, the waveform of the chopper amplifier has a drift in the pressure data. These differences are clearly visible at the end of the measurement. Due to the parallel recording of the force signals, the measurements can be compared directly.

In Fig. 19, no drifts in the force signals could be found. In both Figs. 18 and 19, it can be seen that some production intervals have a different shape. This is an indication of non-optimal production parameters for these test pieces. The automatic classification of production quality is part of the work of Schneider et al. (2016b) and Schneider et al. (2016c). In the following section, the signal fusion with a Kalman filter is described.

\section{Signal optimisation with a Kalman filter}

\subsection{Theory of the Kalman pressure estimation}

Various software filters were investigated in order to improve the signals. One opportunity for the signal optimisation is the Kalman filter. It is assumed here that the chopped pressure signals are applied to the input of the model and transferred into the force signals through the behaviour of the system. This is a kind of real-time data fusion from one pressure and 


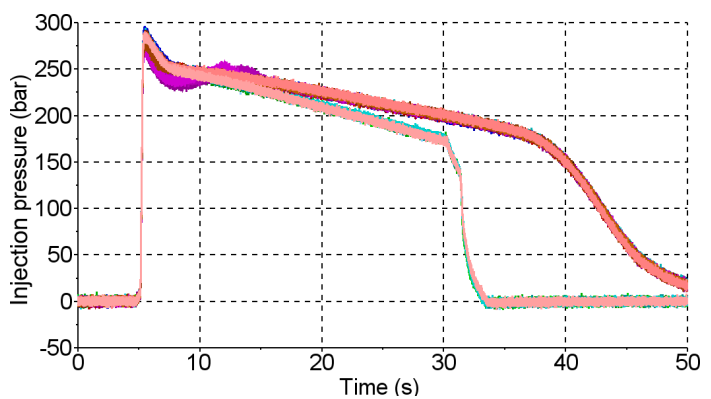

Figure 19. The 25 production intervals measured through the force sensors; the interval time is also $50 \mathrm{~s}$. These production cycles are the same as in Fig. 18.

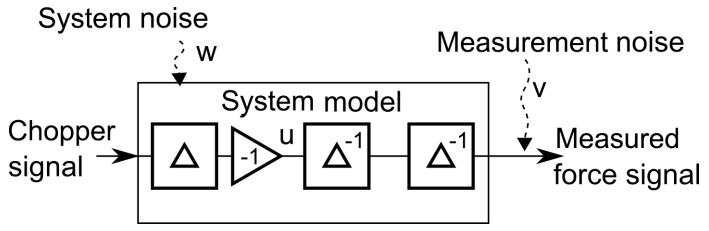

Figure 20. A sketch of the system model with the pressure signal as input and the system, measurement noise and the measured force signal as output.

one force signal. For a better understanding, a sketch of this system with the simplified mathematical operations is shown in Fig. 20. The $\Delta$ block in the figure describes the differentiation of the sampled chopper signal. This operation eliminates the reference voltage in this data series. The next block $(-1)$ inverts the signal. The two $\Delta^{-1}$ blocks describe the two-time integration of the signal to generate the force signal.

The prerequisite for the calculation of the Kalman filter is the state-space representation for the system. Eqs. (7) and (8) are used here as the basic equations for the discrete statespace model similar to Higgins (1974), Welch and Bishop (2000).

$$
\begin{aligned}
\boldsymbol{x}_{n+1} & =\mathbf{A}_{\mathrm{d}} \cdot \boldsymbol{x}_{n}+\mathbf{B}_{\mathrm{d}} \cdot u_{n}+\boldsymbol{w}_{\mathrm{n}} ;(n \in N), \\
y_{n} & =\mathbf{C}_{d} \cdot \boldsymbol{x}_{n}+v_{\mathrm{n}} .
\end{aligned}
$$

The matrices for Eqs. (7) and (8) are simplified and optimised for implementation in an embedded system for integer calculations and higher accuracy:

$\mathbf{A}_{\mathrm{d}}=\left|\begin{array}{ll}1 & 1 \\ 0 & 1\end{array}\right| ; \mathbf{B}_{\mathrm{d}}=\left|\begin{array}{l}0 \\ 1\end{array}\right| ; \mathbf{C}_{d}=|10|$.

The internal states $\boldsymbol{x}_{n+1}$ of the system depend only on the last state from the past $\boldsymbol{x}$ and the input variables $u_{n}$. The index $n$ is the sample number. The system noise $\boldsymbol{w}_{\mathrm{n}}$ and the measurement noise $v_{\mathrm{n}}$ are shown in Fig. 20. Both can be calculated as the standard deviation of the respective signal and were used to model the uncertainty of the system and the measurement.
As Eqs. (1) and (2) show, the reference voltage is still contained in the measured value. One possibility for the removal of the reference voltage is illustrated in Fig. 20. The quantity calculated with Eqs. (10) and (12) represents the input value $u_{n}$ of the state-space model:

$$
\begin{aligned}
u_{s}(t) & =\frac{\Delta u_{s}(t)}{\Delta t}+U_{\mathrm{ref}}, \\
\frac{\Delta^{2} u_{s}(t)}{\Delta t^{2}} & =u_{n} ;(t=n \cdot \Delta t), \\
u_{n} & =\frac{1}{C_{\text {in }}} \cdot \frac{\Delta\left(\int_{t}^{t+\Delta t-t_{\text {reset }}} i(t) d t\right)}{\Delta t^{2}} .
\end{aligned}
$$

In order to transfer the input variable $u_{n}$ into vector form, it is multiplied by the input matrix $\mathbf{B}_{\mathrm{d}}$. The system matrix $\mathbf{A}_{\mathrm{d}}$ describes the double integration of the system model, as can be seen in Fig. 20. Thus, it should be possible to reconstruct from $u_{n}$ the force progression $y_{n}$. The two downstream elements in the system model perform these mathematical operations (see Fig. 21). The Kalman filter created with these assumptions works here as a state observer. It tries to approach the internal states of the system based on an estimate $\hat{u}_{n}$. The filter is represented as a parallel structure of the real system. In Fig. 21, the filter design is displayed. So that the filter can respond to deviations in the system range, the output $y_{n}$ will be constantly compared to the output $\hat{y}_{n}$ of the filter. The difference between these values is used as a correction value for the estimated states $\hat{\mathbf{x}}_{n}$ of the filter:

$\mathbf{K}_{n}=\left|\begin{array}{l}k_{1} \\ k_{2}\end{array}\right|$.

The correction values act through matrix $\mathbf{K}$ on the state vector of the filter. The values $k_{1}$ and $k_{2}$ are the filter coefficients and are adjusted after each iteration of the filter calculation based on the error between $y_{n}$ and $\hat{y}_{n}$. With Eqs. (14) and (15), the required covariance matrix $\mathbf{Q}$ of the system noise $\boldsymbol{\omega}$ and the covariance matrix $\mathbf{R}$ of the measurement noise $v$ can be calculated. In this special case, the matrix $\mathbf{R}$ is only a scalar:

$\mathbf{Q}=\mathbf{B}_{d} \cdot \sigma_{\text {sys }}^{2} \cdot \mathbf{B}_{d}^{T}$,

$\mathbf{R}=\sigma_{\text {meas }}^{2}$.

With the predetermined values, the recursive algorithm can determine the covariance matrices $\mathbf{P}_{n}^{-}$and $\mathbf{P}_{n}$ for the calculation of the estimation error vectors $\boldsymbol{e}^{-}$and $\boldsymbol{e}$ according to Unbehauen (2009), Welch and Bishop (2000):

$\boldsymbol{e}_{n}^{-}=\boldsymbol{x}_{n}-\hat{\boldsymbol{x}}_{n}^{-}$,

$\boldsymbol{e}_{n}=\boldsymbol{x}_{n}-\hat{\boldsymbol{x}}_{n}$.

Matrices and variables with superscript minus are designated as a priori estimations. The a posteriori values are de- 


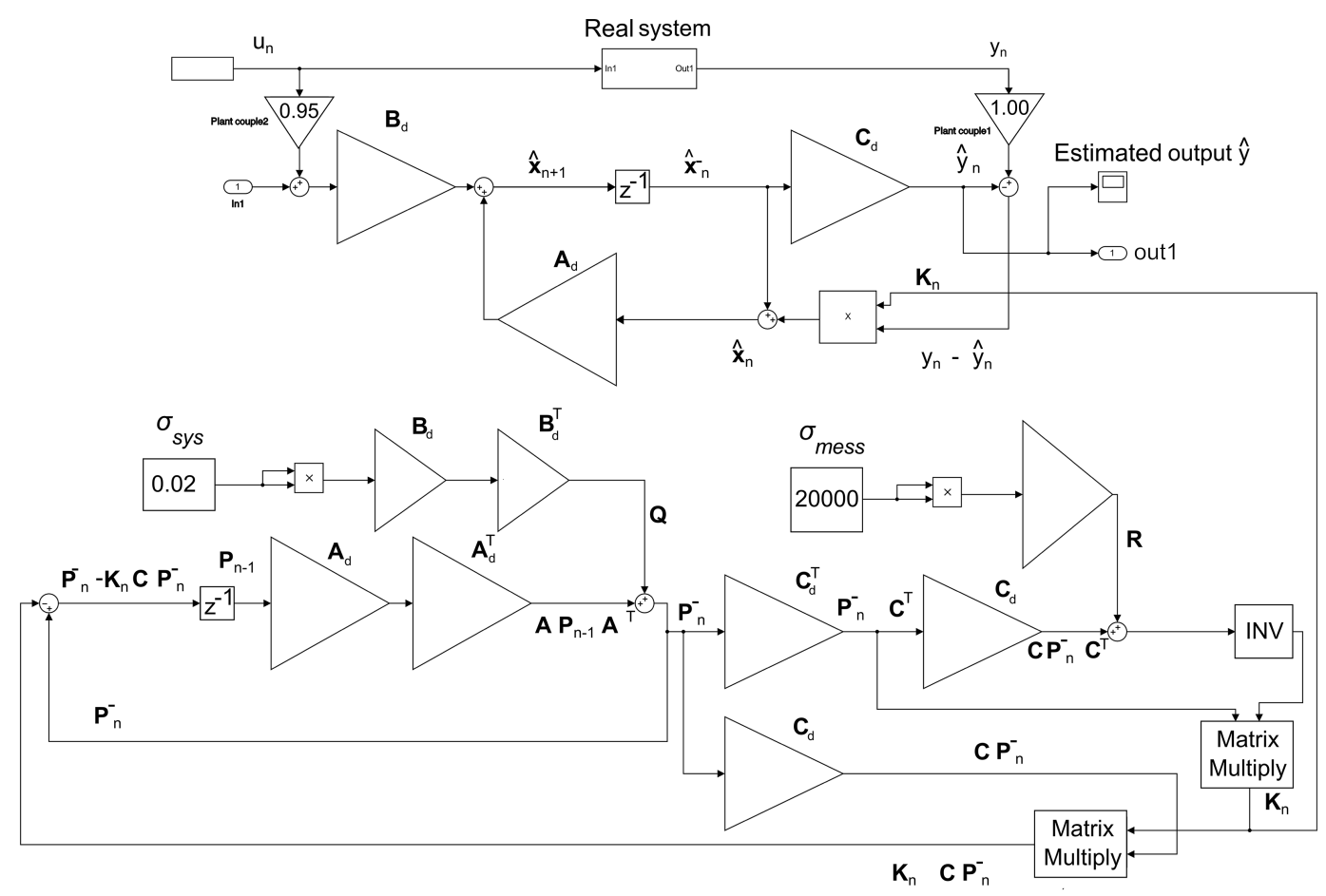

Figure 21. A detailed sketch of the filter design with the addition of the recursive Kalman filter in the lower part (Greifzu, 2015).

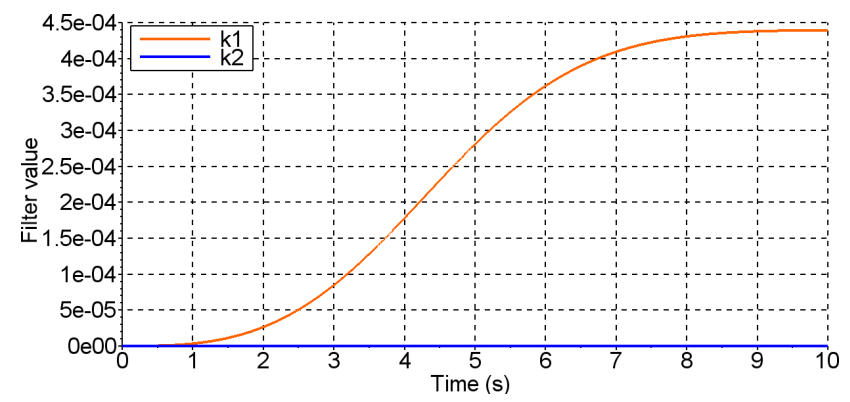

Figure 22. An illustration of the transient phenomenon of the filter coefficients $k_{1}$ and $k_{2}$ of the Kalman filter.

noted without a minus. The following calculation steps run in parallel to the actual filtering process and illustrate the adaptation of the filter coefficients:

$$
\begin{aligned}
\mathbf{P}_{n-1} & =\mathbf{P}_{n-1}^{-}-\mathbf{K}_{n-1} \cdot \mathbf{C} \cdot \mathbf{P}_{n-1}^{-}, \\
\mathbf{P}_{n}^{-} & =\mathbf{A} \cdot \mathbf{P}_{n-1} \cdot \mathbf{A}^{T}+\mathbf{Q}, \\
\mathbf{K}_{n} & =\mathbf{P}_{n}^{-} \cdot \mathbf{C}^{T} \cdot\left(\mathbf{C} \cdot \mathbf{P}_{n}^{-} \cdot \mathbf{C}^{T}+\mathbf{R}\right)^{-1}, \\
\mathbf{P}_{n} & =\mathbf{P}_{n}^{-}-\mathbf{K}_{n} \cdot \mathbf{C} \cdot \mathbf{P}_{n}^{-} .
\end{aligned}
$$

These operations enable the calculation of the internal states $\hat{\boldsymbol{x}}_{n}$ of the filters. The state vector is multiplied by the output matrix $\mathbf{C}$, and as a result the output size $\hat{y}_{n}$ of the filter

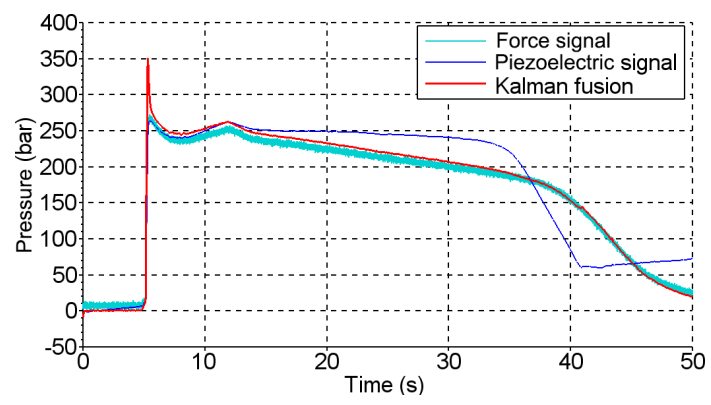

Figure 23. A representation of the force signal (light blue), the piezoelectric signal (blue) and the result of the Kalman filter (red) for one injection moulding cycle.

is created. Figure 21 shows the expanded configuration of the filter design with the addition of the recursive algorithm.

The results of the developed Kalman filter are described in the next section.

\subsection{Results with application of a Kalman filter}

The Kalman filter requires a certain time to settle before it provides useful results from the data fusion. The time needed is approximately $10 \mathrm{~s}$. In Fig. 22, the curve of the filter coefficients during the settling phase is illustrated.

It shows that the coefficient $k_{1}$ only affected the estimates. The value $k_{2}$ has no effect on the state estimations. In order to obtain stable filter coefficients, it is necessary to conduct an 
offline training of the Kalman algorithm. The precomputed coefficients enable the usage without a settling phase. This is a great advantage for implementation in the EDS. The light blue graph in Fig. 23 shows the recorded force signal without filtering. The red curve is the result of the combined sensor signals with the Kalman filter. As one can see, the merged signal has almost no interference and shows good adaptation to the force curve.

The Kalman filter provides good results by reducing the interference level and the offset at the beginning of the force signal. The second point is the elimination of the drift error in the piezoelectric pressure signal. The last feature of the data fusion is the filter phase delay of zero. However, the quality of the data fusion depends on the sensor position and the geometry of the tool cavity. In our case, the data fusion is useful for both force sensors in the ejector.

\section{Conclusions}

Two major advantages of this chopper amplifier are the ground connection of the sensor and the single power supply. The ground connection of the amplifier input results in better shielding of the sensor signals in industrial environments. In special cases, the amplifier can be used with only one cable connection to the piezoelectric sensor. The contact to the ground could be achieved through the tool connection with the metal case of the chopper amplifier.

As can be seen from the results in Sect. 4.2, the interference of the $50 \mathrm{~Hz}$ power grid frequency could be eliminated through the integration of the signal. This voltage drift is likely due to an integration error caused by the interference voltages, an error in the reference voltage and the short "reset" phases during the chopping of the input signal. This error varies in every measurement and depends on the interferences in the chopper amplifier and the chopper signal. The developed chopper amplifier is very robust against external noise. The disturbances during the determination of the signal-to-noise ratio coincide temporally with the start of a permanently installed fan in the climate chamber. The fan motors generate a disturbance peak in another spectral section, which can worsen the SNR by up to $4 \mathrm{~dB}$. In further work, this effect is reduced with better electrical shielding of the electronics.

The calculated threshold of $0.1 \mathrm{pC}$ in Sect. 5.2 is approximately 100 times worse than in commercial charge amplifiers. However, they have considerably larger dimensions and a significantly higher supply voltage; most of the piezoelectric sensors have an accuracy of $10 \mathrm{pC} \mathrm{bar}^{-1}$. For this reason, the question arises of whether it is useful to use highprecision amplifier technology in injection moulding. However, a task for future work is to improve the sensitivity of the chopper amplifier. With dimensions of approximatively 2 to $3 \mathrm{~cm}$, a small PCB design could be achieved, but it would still need to be optimised.
The amplifier was able to be tested for functionality with the measurements in the current manufacturing process. The differences described in Sect. 6.2 between the pressure and force signals over time may be due to the different position of the sensors in the cavity (Schneider and Wenzel, 2014) or the ejection mechanism mounted on the force sensor as described in Sect. 6.1. The developed charge amplifier provides robust and clean results at a sufficient resolution during the production process. With the aid of a Kalman filter, it could be shown that a data fusion between force and pressure signals is possible, and an improvement in the signal quality can be achieved. The noise of the force signal can be reduced. The previously measured drift phenomena in the pressure signal can be eliminated by the data fusion. Furthermore, an advantage of the Kalman filter is that the phase delay of the digital filter is zero.

The result of this investigation is a reduction in the equipment costs for pressure measurement inside an injection moulding tool. With this chopper amplifier and the software filtering of the force and pressure signals, the prices for sensor equipment can be reduced. It could also be a suitable solution for multi-cavity tools. The points on the requirement list from Sect. 2 could be achieved. Data fusion for injection moulding processes is still the subject of current research. With this contribution, it could be shown that the developed amplifier for measuring the charge transfer of piezoelectric sensors has good properties and can be used in embedded systems.

Data availability. The data and results presented in this contribution are part of further research project and special for the used injection moulding machine and tool. Furthermore, the data series itself does not carry any relevants besides demonstrating the chopper charge amplifier and the signal optimisation. Therefore, the data series are not available online, but the authors can provide sample data upon request.

Author contributions. Manuel Schneider and Alexander Jahn designed the schematic circuit diagram, the test environment and the construction of the prototype chopper amplifier. The data analyses, software development and filter design were accomplished by Norbert Greifzu, Manuel Schneider and Norbert Fränzel.

Competing interests. The authors declare that they have no conflict of interest.

Acknowledgements. The authors would like to thank the Thüringer Aufbaubank as a project sponsor for this research. The results of the underlying work were funded by the State of Thuringia and the European Union (ESF), project number 2013 FRG 0130 . 
Edited by: E. Starke

Reviewed by: two anonymous referees

\section{References}

Choi, K.: Measuring of dynamic figures: SNR, THD, SFDR, Department of Computer Science and Engineering, Pennsylvania State University, available at: http://www.cse.psu.edu/ chip/ course/analog/lecture/SFDR1.pdf (last access: 6 May 2017), 2006.

Enz, C. C. and Temes, G. C.: Circuit techniques for reducing the effects of op-amp imperfections:autozeroing, correlated double sampling, and chopper stabilization, P. IEEE 84.11, doi:10.1109/5.542410, 1996.

Gautschi, G.: Piezoelectric Sensorics - Force Strain, Pressure, Acceleration and Acoustic Emission Sensors Materials, 978-3-66204732-3, Springer, 2002.

Greifzu, N.: Entwicklung von Hard- und Software zur Messung von Kraft-, Druck- und Temperatursignalen in Kunststoffspritzgussmaschinen, Master's thesis, University of Applied Sciences Schmalkalden, 2015.

Grellmann, W. and Seidler, S.: Polymer Testing, vol. 2, Carl Hanser Verlag, doi:10.3139/9781569905494, 2013.

Higgins, W. T. J.: A Comparison of Complementary and Kalman Filtering, IEEE Transaction on Aerospace and Electronic Systems, 11.3, 321-325, doi:10.1109/TAES.1975.308081, 1974.

ISO 294-1: Plastics - Injection moulding of test specimens of thermoplastic materials, Beuth Verlag GmbH, 1996.

Jahn, A.: Konzeption, Umsetzung und Test einer analogen Signalaufbereitungsschaltung für piezoelektrische Drucksensoren, Bachelor's thesis, University of Applied Sciences Schmalkalden, 2015.

Kistler Group: Industrial Charge Amplifier for Applications in Manufacturing Type 5073A, available at: https://kistler-embedded.partcommunity.com/3d-cad-models/ FileService/File/kistler/07_electronics/01_charge_amplifiers/ 02_industrial/5073a_english.pdf (last access: 6 May 2017), 2012 .
Priamus System Technologies GmbH: PRIAMUS Easy Ladungsverstärker Typ en 5050A/5050A-M01/5050AM02/5050A-M03, available at: http://www.priamus.com/index. php?option=com_jdownloads \&Itemid $=245 \&$ view $=$ finish \& $\mathrm{cid}=$ $335 \&$ catid $=143 \& \mathrm{~m}=0 \&$ lang=de (last access: 6 May 2017), 2015.

Schneider, M. and Wenzel, A.: Entwurf eines Eingebetteten Diagnosesystems zur Überwachung von Prozessparametern bei Spritzgießen, in: Tag der Forschung, University of Applied Sciences Schmalkalden, doi:10.13140/RG.2.1.4739.5441, 2014.

Schneider, M., Jahn, A., Greifzu, N., and Fränzel, N.: Entwicklung eines unipolaren differentiellen Ladungsverstärkers für die Anwendung in eingebetteten Diagnoseseystemen zur Druckmessung in Spritzgussmaschinen, in: 18. GMA/ITG-Fachtagung Sensoren und Messsysteme, 782-789, doi:10.5162/sensoren2016/P9.2, 2016a.

Schneider, M., Jahn, R., and Wenzel, A.: Erprobung eines echtzeitfähigen Auswertungsalgorithmus zu Bewertung der Fertigungsqualität beim Spritzgießen mit Hilfe eines eingebetteten Diagnosesystems, in: 17. Nachwuchswissenschaftlerkonferenz, University of Applied Sciences Schmalkalden, doi:10.13140/RG.2.1.3829.2887/1, 2016b.

Schneider, M., Walther, C., and Wenzel, A.: Classification of Production Quality in Injection Moulding with an Embedded Diagnostic System Using a Fuzzy Inference System, 26. Workshop Computational Intelligence, 193-203, doi:10.5445/KSP/1000060007, 2016c.

Seefried, A. and Drummer, D.: Prüf- und Probekörperwerkzeuge am Lehrstuhl für Kunststofftechnik, FriedrichAlexander Universität Erlangen-Nürnberg, available at: http://www.lkt.uni-erlangen.de/laboratorien-technika/ fertigungseinrichtungen/Pruef-undProbekoerperwerkzeuge.pdf (last access: 6 May 2017), 2015.

Unbehauen, H.: Regelungstechnik II: Zustandsregelungen, digitale und nichtlineare Regelsysteme, vol. 9, Vieweg+Teubner Verlag, 2009.

Welch, G. and Bishop, G.: An Introduction to the Kalman Filter, University of North Carolina, Department of Computer Science TR 95-041, 2000. 